A solution of the blue vitriol and a caustic soda lye are
then prepared, both at 15 deg. Baumé. The former is placed then prepared, both at 15 deg. Baume. The former is placed
in a copper pan, and heated to about 78 deg. to 100 deg. F.
Room must be left for sufficient alkaline lye to precipitate the solution. The lye is placed in a wooden or iron vessel,
with a plug for running off the lye at pleasure. This vessel is placed over the copper pan, and a s soon as the tempera-
ture is reached, the plug is opened, and the lye is allowed to ture is reached, the plug is opened, and the lye is allowed to
fiow out in a thin stream into the copper solution, stirring fiow out in a thin stream into the copper solution, stirring excess of lyc, must be added, and the temperature must not
be allowed to rise higher, or blackening may ensue. We may ascertain when sufficient lye has been added, by enough lye has been added, the liquid will be as clea as water, and will give no further precipitate with an
addition of more lye. The precipitate, which now apaddition of more
pars green, is taken out and washed, and drained upon
filters till it become a thick paste. It has now to undergo the process of "blueing." FFor this purpose a potash lye is prepared, not perfectly caustic. This is effected by dissolv.
ing in an ordinary caustic lye, at 17 deg. Baumé so much Ing in an ordinary caustic lye, at 17 deg. Baumé, so much
crude potas that ti may mark 22 deg. to 25 deg. Baumé. I
is allowed to settle, and is then fit for use. The blueing i is allowed to settle, and is then fit for use. The blueing is
performed is copper pans, which should be movable.
About 50 lbs, of the green, pan, and about half a small pail of the lye is poured upon it, and immediately stirred well with a wooden spatula. The mixture takes a more blue color, which change goes on for
some time. A small sample is placed upon paper, and the color examined. Another small portion is put in a glass,
little caustic lye is added, stirred up, and compared agai little caustic lye is added, stirred up, and compared again,
and a few drops are placed upon the same paper. If the and a few drops are placed upon the same paper. If the
color of the first sample is satisfactory, or if a further addition of lye produces no more decided blueness, sufficient lye
has been added. If, however, a bluer shade appears in the sample, more lye-say, 14 , pail-is added to the bulk, and stirred up, testing again till the full blue shade has been
reached. The contents of the copper pan are then emptied reached. The contents of the copper pan are then emptied
into a cistern containing a very large excess of water, and
washed repeatedly by decantation so stirring until every trace of caustic lye and alkaline salt is removed. If this is not done the color will become blackened in spots on drying. When the washing is completed, the color is strained through cotton cloths, pressed in cakes about $11 / 2$ inch in thickness,
and then cut into pieces 2 inches square. These are then carefully dried at first at a temperature not exceeding that
of the atmosphere finishing off at about 100 deg. F.; 100 of the atmosphere, finishing off at about 100 deg. F.; 100
lbs. blue vitriol, with 100 lbs. potash, will yield in this man.

WILD YELLOW LAKE

Take any quantity of the clear decoction, dissolve in it a little alum (free from iron), and then precipitate with soda,
carbonate of soda, or, better still, with tin crystals. The carbonate of soda, or, better still, with tin crystals. The
lake is a pure yellow, without any orange cast. The color, however, is not permanent,
to light.-Chemical Revievo.

\section{A NEW OXYGEN RETORT.*}

By William J. Chadwick.

THE oxygen mixture is in the form of perforated cakes,
instead of plugs or cylinders. The shape of the retort is made so that the cake lies upon the bottom, and is only in
contact on that side. The cakes, with the bottom bearing contact on that side. The cakes, with the bottom bearing on the retort, consist simply of manganese, sand, or other
substance containing no chlorate of potash, upon the top of pact cake. The retort consists of two small iron castings, the bottom
one a tlat, circular plate, with two projecting lugs. The
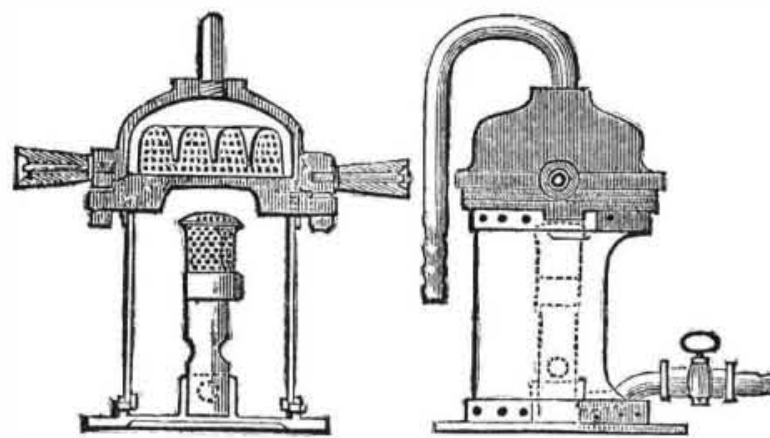

upper portion, which is bell-shaped, is made a little larger
inside than the cakes to be used, and is attached to the botinside than the cakes to be used, and is attached to the bottom portion by means of a bayonet joint, although other
fastenings may be adopted. The gas-tight joint between the fastenings may be adopted. The gas-tight joint between the gether, after having turned them to something like the shape. The stand simply supports the retort over an ordi-
nary Bunsen burner, and may be attached to the top of the
gas-holder, hung upon the side, or placed upon a convenient nasy-holder, hung upon the side, or placed upon a convenient
table, or even on the fioor. From the bent tube on the top portion of the retort an
india-rubber or fiexible pipe conveys the gas direct to the india-rubber or fiexible pipe conveys the gas direct to the
gas-holder. A small wash bottle may be used, or an ordinary back pressure valve, or an ordinary tap. they leave the retort perfectly clean; for, as it is the chlorate of potash that fuses and sticks to the retort, the manganese
or sand which forms the bottom of the cake, and is the only portion in direct contact with the retort, is a sure means of
preventing any sticking. This plan I also adopted in the simply to facilitate the generation of the gas.

\section{PREPARATION OF PURE BISMUTH.}

According to Thirach, pure bismuth may be obtained by the following method: Commercial bismuth is melted under per cent. of sodium carbonate had previously been added the quantity depending upon the amount of impurities in
the bismuth. The resulting regulus is dissolved in the least quantity of nitric acid. The solution is to be mixed with a
concentrated solution of oxalic acid in slight excess, and concentrated solution of oxalic acid in slight excess, and it is separated from the clear liquid by decantation and fil allowed to remain too long in contact with the supernatant

*Read before the Manchester Photographic Society. liquid, it is likely to become contaminated with iron ; but
the subsequent ignition of the dry precipitate again removes arbonic acid. In presence of iron there is formed a bis mutho-ferric oxide, which may be completely separated from
the metal by hydrochloric acid. The chief aim of the author was to devise a process which would remove iron and
silver as completely as possible, but traces of the latter silver as completely as possible, but trac

TREATING LUBRICATING OILS.

HERETOFORE it has been necessary to mix hydrocarbon,
r mineral lubricating oil, with a considerable proportion of oilts, to give it the necessary body or viscidity; bu these fatty oils (both animal and vegetable) are all more or
less oxidizable in the air, producing gummy matters becom-
ing very acid, and if left in warm places on cotton, wool, ng very acid, and if left in warm places on cotton, wool,
clothes, sawdust, or similar material, are exceedingly liable igpontaneous combustion. For lubricating the pistons of marine and stationary, the fatty oils are particularly objec-
tionable, for, in addition to the before-mentioned faults, th high-pressure steam decomposes them into fatty acids, and where the fatty acids corrode most powerfully, and with the glycerin make a kind of soapsuds, producing excessive priming. But Mr. Humfrey, of Chester, England, claims that he is able to produce mineral or hydrocarbonlubricating equired, the viscidity being equal to the best olive, and considerably superior to sperm; while being perfectly neutral,
it cannot act on or corrode the condensers or boilers, nor form concretions in the cavities of the pistons and steam assages; nor does it act on the india rubber valves of the
ir pumps to any injurious extent when used for internal lubricating of steam engines. For ordinary lubricating it is
perfect, as it forms no gum or acid, and it is absolutely safe from spontaneous combustion, with a lubricating power
equal to sperm. The oil treated by his invention is also for all kinds of fast-running fast-running machinery, and the oil to careful fractional distillation, and collects the treatment, instead of agitating the chemicals with the oil by means of paddles, screws, or other mechanical means, as
have heretofore been used, he forces a large stream of compressed air through a plpe at or near the bottom of the vessel as a most thorough and complete agitation, a considerable effect is produced, powerfully aiding the action of the phemicals used; at the same time, the great volume of air and boiling points, the result being lubricating oil possessing more body and higher specific gravity and flash-point tha
any mineral lubricating oils heretofore produced, making it specially adapted for lubricating the pistons, slide valves, and other parts of steam engines, machinery, and other ap paratus. The oil may be produced from coal, shale, peat
bitumen, asphaltum, petroleum, and other oil producers, a is found most economical and convenient.

\section{ESTIMATION OF BORACIC ACID.}

AN easy and practical method for the determination of boracic acid, which has long been a desideratum, appears to method we give in the following: It having been observed
hat the addition of strong alcohol to a solution of boracic acid or a borate, which has been partially precipitated by baryta, causes the complete separation of barium borate would form both a sodium and a barium-salt soluble in alco-
hol. Such an acid was found to be brombydric acid, since $\mathrm{NaBr}$, as well as $\mathrm{BaBr}_{2}$, are easily soluble in 95 per cent. alcohol, and having the additional advantage that sodium hydrated boracic acid

duced into a fiask, colored with a little tincture of litmus and enough bromhydric acid is added to produce an acid
reaction. There is then in solution, sodium bromide, boracic acid, and the excess of bromhydric acid. The fiask is now closed with a doubly perforated cork, carrying a tube acid which might be carried in by the air,* and a small funnel, containing a moistened filter. Cold saturated baryta-
water is passed through the latter into the fiask, until, after gently shaking, the precipitate which forms at first is redis-
solved. Freshly boiled alcohol of 95 per cent. is now poured solved. Freshly boiled alcohol of 95 per cent. is now poured in through the same funnel, so as to produce an alcoholic tube and funnel are removed, and replaced by short glass-
rods, the fiask is slaken and then set aside for 24 hours. The glass rods are then again removed, the potash tube inserted
into one of the perforations, and a syphon tube into the ther, by means of which most of the clear liquid may be
removed. Alcohol of 75 per cent. having been rapidly Alcohol of 75 per cent. having been rapidly
poured in after removal of the syphon, the fask is again
ermetically closed and set aside. When the sediment has been deposited, the liquid is removed in the same manner the sediment, consisting of barium borate transferred to a
tarred filter, and washed with alcohol of 75 per cent., taking care to prevent free access of air, except towards the end. and weighed as bariu

EXPLOSION OF NITRO-HYDROCHLORIC ACID. AT Bow Street Police Court, London, recently, Messrs.
Rouch \& Co., who were represented by their manager, Mr. Rouch \& Co., who were represented by their manager, Mr. ous explosive liquid, and causing damage to a tablecloth and other property in chambers occupied by Captain
Smart, at St. Martin's Chambers, Trafalgar Square.

Mr. Montagu Williams, in support of the summons, said Captain Smart, a gentleman in the military service, but also
well known as an author and novelist. If he had been pursuing his literary occupations at the time of the explosion he
might have been deprived of his eyesight or otherwise might have been deprived of his eyesight or otherwise
injured for life. It appeared that on the morning of the 9th April, a boy delivered at the chambers a small packet, reon the bottle, and the boy was unable to say for whom it wa * The necessity of excluding carbonic acid arises from the subsequen
presence of ree barytain the liquid, which might be converted into car
bonse, and thereby contaminate the precipitated borate. intended. The landlady, Mrs. Billingham, thinking that i must be for Captain Smart, desired the servant to place it on the captain between 10 and 11 at night.

Captain Smart deposed that he saw the packet lying on
is table. He looked at it for a moment, and then sat dow in front of the fire with his back towards the table. Suddena soda-water bottle, and he saw that the packet on the table had disappeared. The room was filled with smoke and a windows, and as both the tablecloth and table appeared to be burning, he procured a wet cloth and took other means to prevent the spreading of fire. He found that the bottle had been splintered to atoms, and the neck of it, with a cork
till in it, was found upon the fioor. Stains caused by the contents of the bottle were to be seen on the carpet, the furniture, and a portion of his own wearing apparel, an the bottle. The label of Messrs. Rouch was still adhering to the bottle. He sent to them for an explanation of the matter, but they treated the inquiry with such nonchalance,
not to say rudeness, that he resolved to place the matter in Mr. G. H. Ogston, analytical chemist, of Mincing Lane
Mrands of his solitors described the mixture as a most dangerous one, stronge than nitric acid, and more destructive than vitriol. It was known as aqua regia, and was a combination of nitric and
hydrochloric acids, and he was surprised that any chemist could have sent out such a preparation without a proper
description of it, and a caution as to its dangerous character written outside.
The defence was that the mixture had been made up from he prescription of a medical man, and, when sent out by the boy, was accompanied by proper instructions, the precription, and the address of the gentleman for whom it was prepared, viz., Major Furlong, who resided in another set of pened that the boy neglected to deliver the prescription, etc.,
and address. With respect to the statement that the clerk nsolence by Mr. Rouch-who said that it was an attempt to extort money, and that he was as "smart" as the complainant-it was alleged that the complainant and his solicitors behaved in a very " bumptious manner," and that Mr. Rouch consequently lost his temper. It was urged that the occurintention the complainant could not be sustained; but Mr. have happened in Major Furlong's chambers with similar or worse results. The defendant said if the instructions had been delivered with the bottle it would have been seen that
he bottle was to be kept in an upright position, and the defendant's boy was called to prove that he lost the paper at
the time, but finding it afterwards, delivered it the next morning.
Mr. Flowers said that there had been great carelessness, $\mathrm{Mr}$. Flowers said that there had been great carelessness,
and fined the defendants 408 . and $\$ 2$ 12s. 6d., the amount of damage done by the explosion.-London Times.

SYNTHESIS OF UREA

IT has been noticed by E. Schmidt (Ber. deut. chem. Gesell. spring of Harkány, when passed into a concentrated aqueous solution of ammonia at $0^{\circ} \mathrm{C}$., is absorbed in large quantities. Ammonium oxysulphocarbamate is formed,

$$
\mathrm{COS}+2 \mathrm{NH}_{3}=\operatorname{COSN}_{2} \mathrm{H}_{6} \text {, }
$$

which after exposure for some time to ordinary tempera$\operatorname{COSN}_{2} \mathrm{H}_{8}=\mathrm{CH}_{4} \mathrm{~N}_{2} \mathrm{O}+\mathrm{H}_{2} \mathrm{~S}$

while another part, combining with water, forms carbonate of ammonia and ammonium sulphide:

$\mathrm{COSN}_{2} \mathrm{H}_{6}+\mathrm{H}_{2} \mathrm{O}=\mathrm{CO}_{2}+2 \mathrm{NH}_{3}+\mathrm{H}_{2} \mathrm{~S}$.

The second reaction is reduced to a minimum if the liquid lecture experiment the with freshly prepared lead oxide; as without the employment of the metalic oxide. Crystals of urea more than an inch in length can readily be formed by
his means. ON FORMATION OF SALTPETRE BY ORGANIC IT is commonly accepted that the nitrates distributed in he ground arise from combustion of ammonia and nitro-

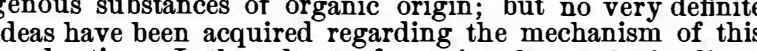
combustion. Is the saltpetre formation the resultof a direct and purely chemical reaction between the oxygen and the
itrogen compounds? Does it occur under the co-operation f organisms which act as ferments, or simultaneously by
oth these processes? "These questions arose," said MM. Schloering and Muntz (in a recent note to the French
Academy), "after M. Pasteur had proved that certain organms, such as the mycoderma of wine and vinegar, hav various organic substances, and are the most active agents for the destruction of organized material which has ceased to live. On the other hand, M. Pasteur shows that organic substances, which are at least as decomposable as those
which form saltpetre in the ground, offer a remarkable resistance to oxygen, when they remain protected from all
organic germs. These two facts led to the supposition that organic germs. These two facts led to the supposition that,
at least in the cases of rapid combustion and formation of it me it may be allowed that ine ind physical force, of consuming nitrogenous substances,

and transforming them into saltpetre."
Starting from this stand-point, the authors made experi-
ments which appear to support the supposition referred to, without absolutely demonstrating its correction

In the course of an investigation as to whether humus must be present in the ground in order that drainage water may be purified, $i . e$., that the substances dissolved in it was filled with 5 kilogrammes of quartz sand that had been strongly heated, and was mixed with 100 grammes of pow-
dered lime. The sand was sprinkled daily with equal quantered lime. The sand was sprinkled daily with equal quan-
tities of drainage water, which was so calculated that the ormation, and the quantity of ammonia in the filtered wate remained unaltered; then saltpetre appeared, and as its quan
tity quickly increased, it was soon evident that the drainage 